Review Article

\title{
An Overall Glance of Evidence Supportive of One-Hour and Two-Hour Postload Plasma Glucose Levels as Predictors of Long-Term Cardiovascular Events
}

\author{
Baldeep K. Mann (D), Janpreet S. Bhandohal ${ }^{D}$, and Jungrak Hong (D) \\ New York City Health and Hospitals/Metropolitan Hospital Center, New York, NY, USA \\ Correspondence should be addressed to Baldeep K. Mann; baldeep.sippi@gmail.com
}

Received 18 August 2019; Accepted 28 November 2019; Published 18 December 2019

Academic Editor: Michael Horowitz

Copyright (c) 2019 Baldeep K. Mann et al. This is an open access article distributed under the Creative Commons Attribution License, which permits unrestricted use, distribution, and reproduction in any medium, provided the original work is properly cited.

\begin{abstract}
This review summarizes the vast literature describing the long-term epidemiological studies with emphasis on postprandial glucose as a stronger predictor of cardiovascular complications as compared to fasting glucose and HbA1c. Many molecular studies also supported this fact by illustrating that postchallenge hyperglycemia is associated with elevated biomarkers of systemic inflammation in the plasma and thus increasing the chances of vascular damage. Large-scale studies have proved that vascular stiffness, brachial-ankle pulse-wave velocity, carotid intima thickness, and left ventricular hypertrophy have been associated with postprandial glucose as compared to fasting glucose or glycosylated hemoglobin.
\end{abstract}

\section{Introduction}

The cardiovascular impact of elevated postprandial glucose has been studied extensively through a significant number of prospective longitudinal, cross-sectional, molecular, and experimental studies in various parts of the world. This concept of relating the postprandial glucose to coronary and cardiovascular events began as early as 1970s with some of the prospective studies at that time. The postprandial state that has been shown to be related to cardiovascular events from time to time was either after 1 hour or 2 hours of ingestion of oral glucose load also known as the oral glucose tolerance test (OGTT). This may be related to the fact that some cases of diabetes (strong risk factor for cardiovascular mortality) can be missed if only fasting blood glucose (FBG) is used in screening [1]. Only few cross-sectional and prospective studies favor glycosylated hemoglobin (HbAlc) being superior to FBG and 2-hour plasma glucose (2 hPG) for prediction of ischemic heart disease [2]. In a cross-sectional study, elevated plasma levels on admission, $\mathrm{HbAlc}$, and FBG were found to have low sensitivity to detect undiagnosed diabetes in patients with acute coronary syndrome as compared to OGTT [3]. The EUROASPIRE IV (the European Action on Secondary Prevention through Intervention to Reduce Events) crosssectional survey of 4004 subjects in 24 European countries showed that OGTT identified a largest number of previously undiagnosed diabetic patients with established coronary artery disease [4]. In a meta-analysis showing that impaired fasting glucose was significantly associated with future risk of coronary heart disease (CHD), the subgroup analyses showed that risk of CHD was only increased in the studies which enrolled participants with increased $2 \mathrm{hPG}$ and not in those which excluded them [5].

\section{Pioneer Epidemiological Studies}

One of the initial investigations showing the relationship of glucose tolerance to the incidence of $\mathrm{CHD}$-associated death and nonfatal myocardial infarction (MI) was two cohort studies consisting of 3,267 and 1,059 Finnish men in 1972 who were followed up over a period of 5 years and the CHD-related death was significantly related to high 1 -h postload blood glucose (1 hPG) level [6]. Later, another 
large prospective study, the Honolulu Heart Program in Hawaii, involving a 20-year follow-up of 457 migrant Japanese patients also found $1 \mathrm{hPG}$ to be positively related to MI-related mortality [7]. Also, in the Honolulu program, odds ratio for $1 \mathrm{hPG}$ in patients with ankle brachial index $<0.9$ was $1.3(p<0.05)$ and found to be predictive of peripheral vascular disease 25 years later [8]. The Helsinki Businessmen prospective study in which 610 men joined a multifactorial primary prevention trial of cardiovascular diseases revealed that $1 \mathrm{hPG}$ was significantly associated with total mortality along with other traditional risk factors (smoking, blood pressure, and cholesterol) after 28 years of follow-up [9]. The evidence strengthened further in the late 1990s with the Chicago Heart association Detection Project in Industry where a 22 year-long longitudinal study of 26,753 nondiabetic men and women showed that $1 \mathrm{hPG}$ level is an independent risk factor for fatal CHD [10].

\section{Subsequent Epidemiological Studies Supportive of Cardiovascular Impact of Postchallenge Hyperglycemia}

US National Library of Medicine (Pub Med.gov) was searched with search terms "post load glucose" and "cardiovascular"; "post load glucose" and "coronary"; "post challenge hyperglycemia" and "cardiovascular"; "post challenge hyperglycemia" and "coronary"; "post prandial glucose" and "cardiovascular"; "post prandial glucose" and "coronary"; "OGTT" and "cardiovascular"; and "OGTT" or "coronary". Various epidemiological studies were found to be conducted from 1999 to 2019 suggesting postprandial glucose as a better predictor of cardiovascular disease independent of FBG that have been enlisted in Table 1. The median follow-up in these trials was 7.2 years with a median number of patients 1425 and median rate of 1.5. Graphical presentation of the rate ratios of studies enlisted in Table 1 has been depicted in Figure 1. Epidemiological data from 20 European studies concluded that $31 \%$ of the diabetic patients with nondiabetic fasting glucose will remain underdiagnosed if only fasting glucose criteria is used and the risk profile of the subjects with impaired fasting glucose depends on 2 hPG levels [42]. Thus, the DECODE study (Diabetes Epidemiology: Collaborative analysis Of Diagnostic criteria in Europe) has emphasized on postchallenge hyperglycemia as the main determinant of risk of cardiovascular disease (CVD) in patients with diabetes [42]. A large prospective Whitehall study involving 17,869 subjects followed up over 33 years established a linear dose-response relationship between postload plasma glucose and CHD mortality risk [14].

\subsection{Postprandial State Enhances Atherosclerosis in Arteries} and Has Detrimental Effects on Myocardial Function. The epidemiological studies were also supported by experimental studies which indicated that the postprandial state may affect atherosclerosis process in the arteries, thereby increasing the coronary vascular events. In European nondiabetic population of 403 subjects, multivariate analysis showed that carotid intima-media thickness was significantly increased in the top $5^{\text {th }}$ quintile of 2 hPG but not the fasting glucose [43]. There was clustering of risk factors such as body mass index, waist to hip ratio, elevated triglycerides, and decreased high-density lipoprotein (HDL-cholesterol) levels in the top quintile of $2 \mathrm{hPG}$ as well [43]. Another cross-sectional study of 356 participants from Pittsburgh site of the Cardiovascular Health Study measured the aortic stiffness through aortic wave pulse velocity, and after controlling age and systolic blood pressure, the strongest predictors of aortic stiffness were heart rate and $2 \mathrm{hPG}(p=0.063)$ [44]. In the RIAD study (Risk factors in Impaired glucose tolerance for Atherosclerosis and Diabetes) intima-media thickness of common carotid arteries that was taken as measure of atherosclerosis was associated with $2 \mathrm{hPG}$ even when $\mathrm{HbA} 1 \mathrm{c}$ was within normal range [45]. This has formed a basis of many studies in 1990s like Diabetes Interventional Study, Kumamoto study, DIGAMI (Diabetes and Insulin-Glucose infusion in Acute Myocardial Infarction) study, and STOP-NIDDM (Study TO Prevent NIDDM) trial to control the postprandial hyperglycemia in order to prevent CVD [45].

In a randomized study where 61 patients with type 2 diabetes were followed up over 24 months and diastolic myocardial dysfunction ( $\left.\mathrm{E}^{\prime}\right)$, intima media thickness and arterial stiffness were significantly higher in patients receiving only conventional insulin therapy (human insulin b.d.) vs. regimens receiving better postmeal glucose control with intensified conventional insulin therapy (lispro at meals and NPH bed time) and supplementary insulin therapy (regular insulin at meals) [46]. In a cross-sectional study from China involving 671 men and 603 women, it was found that the patients with impaired glucose tolerance after glucose challenge have higher brachial-ankle pulse wave velocity/arterial stiffness, are more insulin resistant, and have worse lipid profile [47]. A cross-sectional study of 767 never treated hypertensive subjects showed that $1 \mathrm{hPG}$ of $\geq 155 \mathrm{mg} / \mathrm{dL}$ is a major determinant left ventricular mass index and hence left ventricular hypertrophy in hypertensive patients, that in itself is independent risk factor for cardiovascular morbidity and mortality [48]. In a cross-sectional study including 4938 subjects from China, the ones with impaired glucose tolerance and newly diagnosed diabetes, but not the isolated impaired fasting glucose, had higher brachial-ankle pulse-wave velocity and thus greater arterial stiffness [49]. In a cross-sectional study of 584 newly diagnosed hypertensive individuals, those who had normal glucose tolerance on 2 hPG but had 1 hPG $\geq 155 \mathrm{mg} / \mathrm{dl}$ had higher indices of vascular stiffness (pulse wave velocity, augmentation pressure, and augmentation index) that correlate with the cardiovascular risk profile [50].

3.2. Molecular Studies Relating Postprandial Effect on the Cardiovascular Profile. An experimental study showed that the concentration of soluble forms of the adhesion molecules sE-selectin and sVCAM-1 (vascular cell adhesion molecule1 ), which were hypothesized as early predictors of coronary artery disease, was significantly related to postload glucose 
TABLE 1: Epidemiological studies describing the postprandial hyperglycemia as a predictor of cardiovascular mortality in various parts of the world for the last two decades.

\begin{tabular}{|c|c|c|c|c|c|c|c|c|c|}
\hline No. & Type of study & $\begin{array}{c}\text { Name of the } \\
\text { study }\end{array}$ & $\begin{array}{c}\text { Total } \\
\text { number of } \\
\text { patients }\end{array}$ & $\begin{array}{l}\text { Duration } \\
\text { of follow- } \\
\text { up (years) }\end{array}$ & $\begin{array}{c}\text { Subject } \\
\text { characteristics }\end{array}$ & $\begin{array}{l}\text { Study outcome } \\
\text { measured }\end{array}$ & Rate ratio & $95 \% \mathrm{CI}$ & $p$ value \\
\hline 1 & $\begin{array}{c}\text { Prospective } \\
\text { cohort }\end{array}$ & $\begin{array}{c}\text { Sievers et al., } \\
1999[11]\end{array}$ & 1745 & 10.6 & $\begin{array}{l}\text { Pima Indians } \\
\text { with type } 2 \\
\text { diabetes } \geq 15 \\
\text { years of age }\end{array}$ & $\begin{array}{c}\text { 2-hour } \\
\text { postprandial } \\
\text { glucose }(2 \mathrm{hPG}) \\
\text { levels were } \\
\text { associated with } \\
\text { death rate from } \\
\text { cardiovascular } \\
\text { disease }(\mathrm{CVD})\end{array}$ & $\begin{array}{l}\text { Death } \\
\text { rate } 1.2\end{array}$ & $1.1-1.4$ & 0.007 \\
\hline 2 & $\begin{array}{c}\text { Prospective } \\
\text { cohort }\end{array}$ & $\begin{array}{c}\text { de Vegt et al., } \\
1999 \text { [12] }\end{array}$ & 2363 & 8 & $\begin{array}{l}\text { Dutch subjects } \\
50-75 \text { years } \\
\text { without known } \\
\text { diabetes }\end{array}$ & $\begin{array}{l}\text { Postload glucose } \\
\text { predictive of } \\
\text { increased } \\
\text { cardiovascular } \\
\text { mortality even } \\
\text { within the } \\
\text { nondiabetic } \\
\text { range }\end{array}$ & $\begin{array}{c}\text { Relative } \\
\text { risk (RR) } \\
3.4\end{array}$ & $1.35-8.53$ & $<0.05$ \\
\hline 3 & $\begin{array}{c}\text { Prospective } \\
\text { cohort }\end{array}$ & $\begin{array}{c}\text { Meigs et al., } \\
2002 \text { [13] }\end{array}$ & 3370 & 4 & $\begin{array}{l}\text { Subjects from the } \\
\text { Framingham } \\
\text { offspring study } \\
\text { without clinical } \\
\text { CVD or } \\
\text { medication- } \\
\text { treated diabetes }\end{array}$ & $\begin{array}{c}2 \mathrm{hPG} \text { is } \\
\text { associated with } \\
\text { cardiovascular } \\
\text { events }\end{array}$ & RR 1.14 & $1.02-1.27$ & $\begin{array}{c}\text { Not } \\
\text { available }\end{array}$ \\
\hline 4 & $\begin{array}{c}\text { Prospective } \\
\text { cohort }\end{array}$ & $\begin{array}{c}\text { Brunner et al., } \\
2006 \text { [14] }\end{array}$ & 17869 & 33 & $\begin{array}{l}\text { London-based } \\
\text { male civil } \\
\text { servants aged } \\
40-64 \text { years } \\
\text { excluding those } \\
\text { with known } \\
\text { diabetes and with } \\
\text { missing glucose } \\
\text { measurements }\end{array}$ & $\begin{array}{c}2 \text { hPG associated } \\
\text { with coronary } \\
\text { heart disease }\end{array}$ & $\begin{array}{l}\text { Hazard } \\
\text { ratio } \\
\text { (HR) } 3.62\end{array}$ & $2.34-5.56$ & $\begin{array}{c}\text { Not } \\
\text { available }\end{array}$ \\
\hline 5 & $\begin{array}{c}\text { Prospective } \\
\text { cohort }\end{array}$ & $\begin{array}{l}\text { Meisinger et al., } \\
2006 \text { [15] }\end{array}$ & 1160 & 30 & $\begin{array}{c}\text { Randomly } \\
\text { selected } 40-59 \\
\text { year non-diabetic } \\
\text { German subjects }\end{array}$ & $\begin{array}{l}\text { 1-hour postload } \\
\text { glucose (1 hPG) } \\
\text { associated with } \\
\text { all-cause } \\
\text { mortality }\end{array}$ & HR 1.49 & $1.17-1.88$ & $\begin{array}{c}\text { Not } \\
\text { available }\end{array}$ \\
\hline 6 & $\begin{array}{c}\text { Prospective } \\
\text { cohort } \\
(1974-1979)\end{array}$ & $\begin{array}{l}\text { Nigam et al., } \\
2007 \text { [16] }\end{array}$ & 1691 & 14.7 & $\begin{array}{c}\text { Patients with } \\
\text { coronary artery } \\
\text { disease (CAD) } \\
\text { who were } \\
\text { enrolled at } 15 \\
\text { centers } \\
\text { throughout } \\
\text { North America }\end{array}$ & $\begin{array}{c}\text { Postprandial } \\
\text { hyperglycemia } \\
\text { was not } \\
\text { associated with } \\
\text { cardiovascular } \\
\text { mortality in } \\
\text { patients with } \\
\text { undiagnosed } \\
\text { diabetes }\end{array}$ & HR 0.89 & $0.59-1.36$ & $\begin{array}{c}\text { Not } \\
\text { available }\end{array}$ \\
\hline 7 & $\begin{array}{c}\text { Prospective } \\
\text { cohort }\end{array}$ & $\begin{array}{c}\text { Chien et al., } \\
2008 \text { [17] }\end{array}$ & 2165 & 10.5 & $\begin{array}{c}\text { Chinese subjects } \\
\text { in Taiwan aged } \\
\geq 35 \text { years }\end{array}$ & $\begin{array}{l}\text { Postchallenge } \\
\text { glucose was } \\
\text { associated with } \\
\text { major } \\
\text { cardiovascular } \\
\text { events } \\
\end{array}$ & RR 2.05 & $1.23-3.42$ & $\leq 0.001$ \\
\hline 8 & $\begin{array}{c}\text { Prospective } \\
\text { cohort }\end{array}$ & $\begin{array}{l}\text { Sarwar et al., } \\
2010 \text { [18] }\end{array}$ & 18569 & 23.5 & $\begin{array}{l}\text { Iceland subjects } \\
\text { without history } \\
\text { of diabetes and } \\
\text { myocardial } \\
\text { infarction (MI) }\end{array}$ & $\begin{array}{l}\text { Postload glucose } \\
\text { associated with } \\
\text { coronary heart } \\
\text { disease }\end{array}$ & HR 1.03 & $1.01-1.05$ & $\begin{array}{c}\text { Not } \\
\text { available }\end{array}$ \\
\hline
\end{tabular}


TABle 1: Continued.

\begin{tabular}{|c|c|c|c|c|c|c|c|c|c|}
\hline No. & Type of study & $\begin{array}{c}\text { Name of the } \\
\text { study }\end{array}$ & $\begin{array}{c}\text { Total } \\
\text { number of } \\
\text { patients }\end{array}$ & $\begin{array}{l}\text { Duration } \\
\text { of follow- } \\
\text { up (years) }\end{array}$ & $\begin{array}{c}\text { Subject } \\
\text { characteristics }\end{array}$ & $\begin{array}{l}\text { Study outcome } \\
\text { measured }\end{array}$ & Rate ratio & $95 \% \mathrm{CI}$ & $p$ value \\
\hline 9 & $\begin{array}{l}\text { Meta-analysis } \\
\text { of } 26 \text { western } \\
\text { prospective } \\
\text { cohort studies }\end{array}$ & $\begin{array}{l}\text { Sarwar et al., } \\
2010 \text { [18] }\end{array}$ & 12652 & $\begin{array}{c}\text { Not } \\
\text { applicable }\end{array}$ & Not applicable & $\begin{array}{l}\text { Postload glucose } \\
\text { associated with } \\
\text { coronary heart } \\
\text { disease }\end{array}$ & RR 1.05 & $1.03-1.07$ & $\begin{array}{c}\text { Not } \\
\text { available }\end{array}$ \\
\hline 10 & $\begin{array}{c}\text { Prospective } \\
\text { cohort }\end{array}$ & $\begin{array}{l}\text { Kitada et al., } \\
2010 \text { [19] }\end{array}$ & 422 & 2 & $\begin{array}{l}\text { Acute MI (AMI) } \\
\text { Japanese patients }\end{array}$ & $\begin{array}{l}2 \mathrm{hPG} \text { was the } \\
\text { only } \\
\text { independent } \\
\text { predictor of } \\
\text { long-term major } \\
\text { adverse } \\
\text { cardiovascular } \\
\text { events (MACE) } \\
\text { two years after } \\
\text { AMI }\end{array}$ & $\begin{array}{c}\text { Odds } \\
\text { ratio } \\
(\mathrm{OR}) 1.85\end{array}$ & $1.07-3.21$ & 0.028 \\
\hline 11 & Case control & $\begin{array}{l}\text { Shimabukuro } \\
\text { et al., } 2011 \text { [20] }\end{array}$ & 287 & $\begin{array}{c}\text { Not } \\
\text { applicable }\end{array}$ & $\begin{array}{l}\text { Japanese who } \\
\text { visited the } \\
\text { university } \\
\text { hospital to be } \\
\text { checked for } \\
\text { glucose } \\
\text { intolerance or } \\
\text { known type } 2 \\
\text { diabetes were } \\
\text { consecutively } \\
\text { recruited }\end{array}$ & $\begin{array}{l}\text { Left ventricle } \\
\text { dysfunction } \\
\text { associated with } \\
\text { impaired } \\
\text { glucose } \\
\text { tolerance }\end{array}$ & OR 3.43 & $1.09-11.2$ & 0.037 \\
\hline 12 & $\begin{array}{c}\text { Prospective } \\
\text { cohort } \\
\text { (HEART2D } \\
\text { trial) }\end{array}$ & $\begin{array}{c}\text { Raz et al., } 2011 \\
{[21]}\end{array}$ & 1115 & 2.7 & $\begin{array}{c}\text { Patients with } \\
\text { type } 2 \text { diabetes } \\
\text { who survived of } \\
\text { AMI }\end{array}$ & $\begin{array}{l}\text { Patients using } \\
\text { insulin targeting } \\
\text { the postprandial } \\
\text { versus fasting } \\
\text { hyperglycemia } \\
\text { had lower } \\
\text { cardiovascular } \\
\text { events }\end{array}$ & HR 0.69 & $0.49-0.96$ & 0.029 \\
\hline 13 & $\begin{array}{c}\text { Large } \\
\text { prospective } \\
\text { cohort } \\
\text { (EpiDREAM } \\
\text { study) }\end{array}$ & $\begin{array}{l}\text { Anand et al., } \\
2012 \text { [22] }\end{array}$ & 18,990 & 3.5 & $\begin{array}{c}\text { 30-85 years } \\
\text { multiethnic } \\
\text { patients from } 21 \\
\text { countries who } \\
\text { had impaired } \\
\text { glucose tolerance } \\
\text { (IGT) and } \\
\text { impaired fasting } \\
\text { glucose (IFG) } \\
\text { levels }\end{array}$ & $\begin{array}{l}\text { 2-hour post- } \\
\text { OGTT glucose } \\
\text { associated with } \\
\text { increase in risk } \\
\text { of } \\
\text { cardiovascular } \\
\text { events or death }\end{array}$ & HR 1.17 & $1.13-1.22$ & $\begin{array}{c}\text { Not } \\
\text { available }\end{array}$ \\
\hline 14 & $\begin{array}{c}\text { Prospective } \\
\text { cohort }\end{array}$ & $\begin{array}{l}\text { Tamita et al., } \\
2012 \text { [23] }\end{array}$ & 275 & 5.3 & $\begin{array}{c}\text { Japanese subjects } \\
\text { with AMI }\end{array}$ & $\begin{array}{c}\text { Abnormal } \\
\text { glucose } \\
\text { tolerance } \\
\text { associated with } \\
\text { MACE } \\
\end{array}$ & HR 2.65 & $1.37-5.15$ & 0.004 \\
\hline 15 & $\begin{array}{c}\text { Prospective } \\
\text { cohort }\end{array}$ & $\begin{array}{l}\text { Furtado de } \\
\text { Souza et al., } \\
2012 \text { [24] }\end{array}$ & 148 & $\begin{array}{l}36 \pm 14 \\
\text { months }\end{array}$ & $\begin{array}{l}\text { Brazilian subjects } \\
\text { undergoing } \\
\text { diabetes } \\
\text { screening } \\
\text { attending a } \\
\text { primary care unit }\end{array}$ & $\begin{array}{l}\text { 2-hour OGTT } \\
\text { results were } \\
\text { associated with } \\
\text { CVD }\end{array}$ & OR 1.013 & $1.002-1.025$ & 0.024 \\
\hline
\end{tabular}


TABle 1: Continued.

\begin{tabular}{|c|c|c|c|c|c|c|c|c|c|}
\hline No. & Type of study & $\begin{array}{c}\text { Name of the } \\
\text { study }\end{array}$ & $\begin{array}{l}\text { Total } \\
\text { number of } \\
\text { patients }\end{array}$ & $\begin{array}{l}\text { Duration } \\
\text { of follow- } \\
\text { up (years) }\end{array}$ & $\begin{array}{c}\text { Subject } \\
\text { characteristics }\end{array}$ & $\begin{array}{l}\text { Study outcome } \\
\text { measured }\end{array}$ & Rate ratio & $95 \% \mathrm{CI}$ & $p$ value \\
\hline 16 & $\begin{array}{l}9 \text { Finnish and } \\
\text { Swedish } \\
\text { prospective } \\
\text { cohort }\end{array}$ & $\begin{array}{c}\text { Ning et al., } 2012 \\
{[25]}\end{array}$ & $\begin{array}{l}3743 \text { men } \\
\text { and } 3916 \\
\text { women }\end{array}$ & 16.4 & $\begin{array}{c}25 \text { to } 90 \text { years } \\
\text { who had fasting } \\
\text { plasma glucose } \\
(\mathrm{FPG}) \\
<6.1 \mathrm{mmol} / 1 \text { and } \\
2 \mathrm{~h} \\
\mathrm{PG}<7.8 \mathrm{mmol} / \mathrm{l} \\
\text { and free of CVD }\end{array}$ & $\begin{array}{c}2 \text { hPG associated } \\
\text { with coronary } \\
\text { heart disease }\end{array}$ & $\begin{array}{l}\text { HR } 1.13 \\
\text { in men; } \\
1.33 \text { in } \\
\text { women }\end{array}$ & $\begin{array}{l}0.93-1.37 \text { in } \\
\text { men; } \\
0.83-2.13 \text { in } \\
\text { women }\end{array}$ & $\begin{array}{c}\text { Not } \\
\text { available }\end{array}$ \\
\hline 17 & $\begin{array}{l}\text { Prospective } \\
\text { cohort }\end{array}$ & $\begin{array}{c}\text { Henareh and } \\
\text { Agewall, } 2012 \\
{[26]}\end{array}$ & 123 & $6.03 \pm 1.36$ & $\begin{array}{c}\text { Swedish subjects } \\
\text { aged } 31-80 \text { years } \\
\text { who had suffered } \\
\text { a previous MI }\end{array}$ & $\begin{array}{l}2 \mathrm{hPG} \text { was a } \\
\text { significant } \\
\text { predictor of } \\
\text { cardiovascular } \\
\text { death, recurrent } \\
\text { MI, and unstable } \\
\text { angina pectoris }\end{array}$ & HR 1.27 & $1.00-1.62$ & $<0.05$ \\
\hline 18 & $\begin{array}{l}\text { Prospective } \\
\text { cohort }\end{array}$ & $\begin{array}{c}\text { Silbernagel } \\
\text { et al., } 2012 \text { [27] }\end{array}$ & 1772 & $7.7 \pm 2.0$ & $\begin{array}{c}\text { German } \\
\text { nondiabetic } \\
\text { subjects who } \\
\text { were referred for } \\
\text { angiography and } \\
\text { whose FPG was } \\
<126 \mathrm{mg} / \mathrm{dl} \\
\text { underwent } \\
\text { OGTT }\end{array}$ & $\begin{array}{l}\text { Postchallenge } \\
\text { glucose } \\
\text { undetected by } \\
\text { fasting glucose } \\
\text { and glycated } \\
\text { hemoglobin } \\
\text { independently } \\
\text { predicted the } \\
\text { cardiovascular } \\
\text { mortality }\end{array}$ & HR 1.57 & $1.02-2.43$ & 0.041 \\
\hline 19 & $\begin{array}{l}\text { Cross sectional } \\
\text { (second strong } \\
\text { heart study) }\end{array}$ & $\begin{array}{l}\text { Capaldo et al., } \\
2013 \text { [28] }\end{array}$ & 562 & $\begin{array}{c}\text { Not } \\
\text { applicable }\end{array}$ & $\begin{array}{c}\text { American } \\
\text { nondiabetic and } \\
\text { nonhypertensive } \\
\text { Indians of } 45-74 \\
\text { years of age }\end{array}$ & $\begin{array}{l}\text { Both higher IFG } \\
\text { and IGT levels } \\
\text { rather than only } \\
\text { IFG associated } \\
\text { with left } \\
\text { ventricular } \\
\text { hypertrophy } \\
\end{array}$ & OR 9.76 & $2.03-46.79$ & 0.004 \\
\hline 20 & $\begin{array}{l}\text { Prospective } \\
\text { cohort }\end{array}$ & $\begin{array}{c}\text { Barzin et al., } \\
2013 \text { [29] }\end{array}$ & 3794 & 8 & $\begin{array}{c}\text { Tehran urban } \\
\text { subjects aged } \geq 40 \\
\text { years without } \\
\text { history of } \\
\text { diabetes or CVD }\end{array}$ & $\begin{array}{c}\text { Isolated } \\
\text { postchallenge } \\
\text { hyperglycemia } \\
\text { associated with } \\
\text { cardiovascular } \\
\text { events } \\
\end{array}$ & HR 1.77 & $1.19-2.64$ & 0.005 \\
\hline 21 & Cross sectional & $\begin{array}{c}\text { Yang et al., } 2013 \\
{[30]}\end{array}$ & 6040 & $\begin{array}{c}\text { Not } \\
\text { applicable }\end{array}$ & $\begin{array}{l}\text { Chinese } \\
\text { prediabetic } \\
\text { subjects }\end{array}$ & $\begin{array}{l}\text { CVD events } \\
\text { associated with } \\
\text { IGT levels } \\
\text { compared to } \\
\text { IFG levels }\end{array}$ & OR 2.88 & $1.36-6.01$ & 0.0059 \\
\hline 22 & $\begin{array}{c}\text { Prospective } \\
\text { cohort }\end{array}$ & $\begin{array}{c}\text { Kuramitsu } \\
\text { et al., } 2013 \text { [31] }\end{array}$ & 828 & 4.3 & $\begin{array}{l}\text { Japanese patients } \\
\text { of stable angina } \\
\text { undergoing } \\
\text { percutaneous } \\
\text { intervention } \\
(\mathrm{PCI})\end{array}$ & $\begin{array}{l}\text { Postchallenge } \\
\text { hyperglycemia } \\
\text { was associated } \\
\text { with MACE }\end{array}$ & HR 1.62 & $1.07-2.53$ & 0.023 \\
\hline 23 & $\begin{array}{l}\text { Finnish } \\
\text { diabetes } \\
\text { prevention } \\
\text { prospective } \\
\text { cohort study }\end{array}$ & $\begin{array}{l}\text { Lind et al., } 2014 \\
{[32]}\end{array}$ & 504 & 13 & $\begin{array}{c}\text { Finnish } \\
\text { individuals with } \\
\text { IGT were } \\
\text { followed up with } \\
\text { yearly OGTT, } \\
\text { FPG, and HbA1c }\end{array}$ & $\begin{array}{l}2 \mathrm{hPG} \text { was } \\
\text { associated with } \\
\text { CVD events }\end{array}$ & HR 2.19 & $1.51-3.18$ & $\leq 0.001$ \\
\hline
\end{tabular}


TABle 1: Continued.

\begin{tabular}{|c|c|c|c|c|c|c|c|c|c|}
\hline No. & Type of study & $\begin{array}{c}\text { Name of the } \\
\text { study }\end{array}$ & $\begin{array}{l}\text { Total } \\
\text { number of } \\
\text { patients }\end{array}$ & $\begin{array}{l}\text { Duration } \\
\text { of follow- } \\
\text { up (years) }\end{array}$ & $\begin{array}{c}\text { Subject } \\
\text { characteristics }\end{array}$ & $\begin{array}{l}\text { Study outcome } \\
\text { measured }\end{array}$ & Rate ratio & $95 \% \mathrm{CI}$ & $p$ value \\
\hline 24 & $\begin{array}{l}\text { Prospective } \\
\text { cohort }\end{array}$ & $\begin{array}{l}\text { Ritsinger et al., } \\
2015 \text { [33] }\end{array}$ & $\begin{array}{l}167 \text { AMI } \\
\text { patients } \\
\text { and } 184 \\
\text { controls }\end{array}$ & 10 & $\begin{array}{l}\text { Swedish patients } \\
\text { up to } 80 \text { years } \\
\text { with AMI } \\
(n=167) \text { and } \\
\text { healthy controls } \\
(n=184) \text { with no } \\
\text { previously } \\
\text { known diabetes }\end{array}$ & $\begin{array}{l}\text { Patient with } \\
\text { AMI having } \\
\text { abnormal } \\
\text { glucose } \\
\text { tolerance after } \\
\text { an OGTT } \\
\text { performed at the } \\
\text { time of } \\
\text { discharge had } \\
\text { higher } \\
\text { cardiovascular } \\
\text { mortality }\end{array}$ & HR 2.3 & $1.24-4.25$ & 0.008 \\
\hline $25 a$ & $\begin{array}{l}\text { Yorkshire } \\
\text { retrospective } \\
\text { cohort }\end{array}$ & $\begin{array}{c}\text { George et al., } \\
2015 \text { [34] }\end{array}$ & 768 & 3 & $\begin{array}{l}\text { Patients without } \\
\text { pre-existing } \\
\text { diabetes mellitus } \\
\text { post-MI }\end{array}$ & $\begin{array}{c}\text { IGT associated } \\
\text { with increased } \\
\text { incidence of } \\
\text { MACE } \\
\end{array}$ & HR 1.54 & $1.06-2.24$ & 0.024 \\
\hline $25 b$ & $\begin{array}{l}\text { Yorkshire } \\
\text { retrospective } \\
\text { cohort study }\end{array}$ & $\begin{array}{c}\text { George et al., } \\
2015 \text { [34] }\end{array}$ & 768 & 3 & $\begin{array}{l}\text { Patients without } \\
\text { pre-existing } \\
\text { diabetes mellitus } \\
\text { post-MI }\end{array}$ & $\begin{array}{c}\text { Newly } \\
\text { diagnosed } \\
\text { diabetes } \\
\text { associated with } \\
\text { increased } \\
\text { incidence of } \\
\text { MACE }\end{array}$ & HR 2.15 & $1.42-3.24$ & 0.003 \\
\hline 26 & $\begin{array}{l}\text { Prospective } \\
\text { cohort }\end{array}$ & $\begin{array}{l}\text { Faghihi- } \\
\text { Kashani et al., } \\
2016 \text { [35] }\end{array}$ & 2607 & 7.2 & $\begin{array}{c}\text { Patients of type } 2 \\
\text { diabetes mellitus } \\
\text { in Tehran }\end{array}$ & $\begin{array}{c}2 \text { hPG was } \\
\text { associated with } \\
\text { high incidence } \\
\text { of coronary } \\
\text { heart disease }\end{array}$ & HR 1.64 & $1.03-2.61$ & $\begin{array}{c}\text { Not } \\
\text { available }\end{array}$ \\
\hline 27 & $\begin{array}{l}\text { Prospective } \\
\text { cohort }\end{array}$ & $\begin{array}{c}\text { Shahim et al., } \\
2017 \text { [36] }\end{array}$ & 4004 & 2 & $\begin{array}{c}24 \text { European } \\
\text { subjects aged } \\
\geq 18-80 \text { years } \\
\text { hospitalized for a } \\
\text { first or recurrent } \\
\text { CAD event }\end{array}$ & $\begin{array}{c}2 \text { hPG associated } \\
\text { with } \\
\text { cardiovascular } \\
\text { events }\end{array}$ & HR 1.38 & $1.07-1.78$ & 0.01 \\
\hline 28 & $\begin{array}{l}\text { Prospective } \\
\text { cohort }\end{array}$ & $\begin{array}{l}\text { Nielsen et al., } \\
2017 \text { [37] }\end{array}$ & 4934 & 27 & $\begin{array}{l}\text { Swedish subjects } \\
\text { without diabetes }\end{array}$ & $\begin{array}{c}1 \mathrm{hPG} \text { predicted } \\
\text { the } \\
\text { cardiovascular } \\
\text { death }\end{array}$ & HR 1.09 & $1.01-1.17$ & 0.02 \\
\hline 29 & $\begin{array}{c}\text { Prospective } \\
\text { cohort }\end{array}$ & $\begin{array}{c}\text { Chattopadhyay } \\
\text { et al., February } \\
2018 \text { [38] }\end{array}$ & 674 & 4 & $\begin{array}{c}\text { Post-MI } \\
\text { survivors without } \\
\text { known diabetes } \\
\text { in England and } \\
\text { Wales }\end{array}$ & $\begin{array}{l}\text { Only } 2 \text { hPG } \\
\text { predicted } \\
\text { MACE }\end{array}$ & HR 1.12 & $1.04-1.20$ & $\leq 0.001$ \\
\hline 30 & $\begin{array}{l}\text { Retrospective } \\
\text { cohort }\end{array}$ & $\begin{array}{l}\text { Chattopadhyay } \\
\text { et al., August } \\
2018 \text { [39] }\end{array}$ & 1056 & $\begin{array}{c}40.8 \\
\text { months }\end{array}$ & $\begin{array}{c}\text { Acute coronary } \\
\text { event survivors } \\
\text { without known } \\
\text { diabetes mellitus } \\
\text { who had FBG } \\
\text { and } 2 \text { hPG } \\
\text { measured } \\
\text { predischarge }\end{array}$ & $\begin{array}{c}2 \mathrm{hPG} \\
\text { independently } \\
\text { predicted } \\
\text { MACE }\end{array}$ & HR 1.091 & $1.043-1.142$ & $\leq 0.001$ \\
\hline 31 & $\begin{array}{c}\text { Cross sectional } \\
\text { (CATAMERI } \\
\text { study) }\end{array}$ & $\begin{array}{l}\text { Fiorentino } \\
\text { et al., } 2019 \text { [40] }\end{array}$ & 1010 & $\begin{array}{c}\text { Not } \\
\text { applicable }\end{array}$ & $\begin{array}{c}\text { Nondiabetic } \\
\text { Caucasian } \\
\text { individuals with } \\
\text { hbA1c }<5.7 \%\end{array}$ & $\begin{array}{l}1 \mathrm{hPG} \text { during } \\
\text { OGTT } \geq 155 \mathrm{mg} / \\
\text { dl associated } \\
\text { with CAD }\end{array}$ & OR 6.16 & $1.05-36.32$ & 0.04 \\
\hline 32 & $\begin{array}{l}\text { Retrospective } \\
\text { cohort }\end{array}$ & $\begin{array}{l}\text { Chattopadhyay } \\
\text { et al., } 2019 \text { [41] }\end{array}$ & 1056 & 2.8 & $\begin{array}{l}\text { MI survivors in } \\
\text { East yorkshire } \\
\text { and North } \\
\text { Lincolnshire }\end{array}$ & $\begin{array}{c}2 \text { hPG predicted } \\
\text { MACE-free } \\
\text { survival }\end{array}$ & HR 1.16 & $1.07-1.26$ & $\leq 0.001$ \\
\hline
\end{tabular}




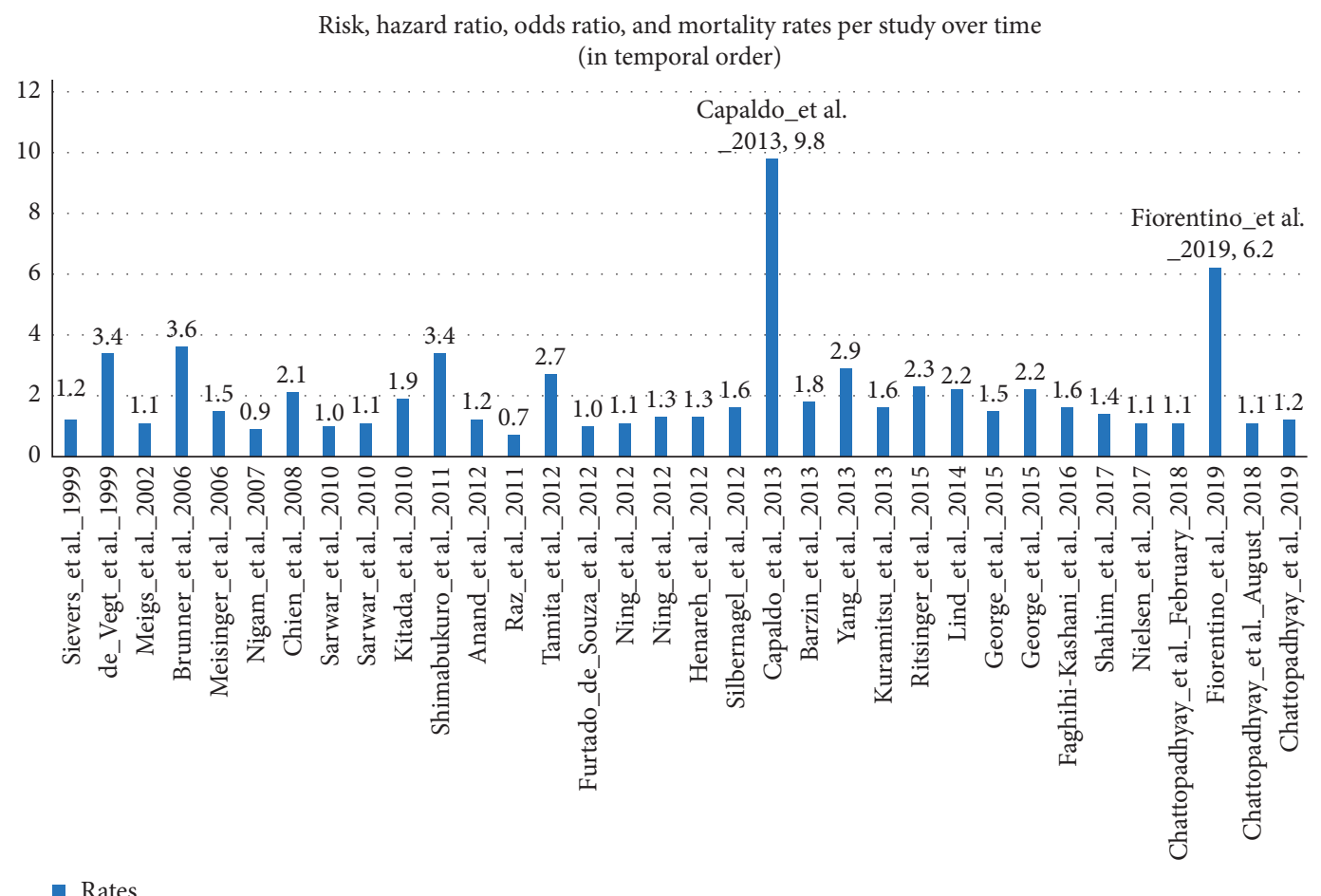

FiguRE 1: Graphical presentation of risks, hazard ratios, odds ratios, or mortality rates per study enlisted in Table 1 in temporal order.

concentration in 78 men with symptoms of angina and positive exercise stress test who presented for coronary arteriography [51]. In a large observational study, it was found that the oral glucose tolerance test increases biomarkers of systemic inflammation such as hsCRP, IL-6, TNF-alpha, sICAM-1, sVCAM-1, and sE-selectin [52]. Postchallenge hyperglycemia is associated with increased generation of asymmetric dimethylarginine and reactive oxygen species that lead to decreased nitric oxide synthesis which decreases endothelial-dependent flow-mediated dilation and hence decreased vascular function [53]. In a CODAM (Cohort on Diabetes and Atherosclerosis Maastricht) study, $\alpha$-dicarbonyl concentrations are correlated with glucose levels during OGTT, not with FBG or HbA1c in individuals with impaired glucose metabolism and type 2 diabetes which are associated with more vascular complications [54].

3.3. Clustering of Cardiovascular Risk Factors with Elevated Postload Glucose. A cross-sectional study of 1475 subjects of Arian ethnicity showed that cardiovascular risk factors like age, body mass index, waist circumference, blood pressure, and lipid profile (not low-HDL cholesterol) were significantly higher in normal glucose tolerance subjects with $1 \mathrm{hPG}$ $>155 \mathrm{mg} / \mathrm{dl}$ [55]. In a multicenter cross-sectional GENFIEV (GENetics, PHYsiopathology, and EVolution of Type 2 diabetes) study, $1 \mathrm{hPG}>155 \mathrm{mg} / \mathrm{dl}$ showed lower insulin sensitivity, impaired $\beta$-cell function, and worse cardiovascular risk profile (glycosylated hemoglobin, blood pressure, lowdensity lipoprotein cholesterol, and triglyceride) [56]. In the 2005-2014 National Health and Nutrition examination survey, a cross-sectional survey of 3644 adults with prediabetes based on FBG and HbAlc, 6.9\% were detected to have diabetes based on $2 \mathrm{hPG}$ and were more likely to have hypertension, higher triglycerides, lower high-density lipoprotein cholesterol, and higher albuminuria [57]. In a DICAMANO study of 447 overweight/obese subjects with FBG $\leq 5.5 \mathrm{mmol} / \mathrm{l}(99 \mathrm{mg} / \mathrm{dl})$ and $\mathrm{BMI} \geq 25 \mathrm{~kg} / \mathrm{m}^{2}$ who underwent a $75 \mathrm{~g}$ OGTT after multivariable adjustment for FBG, smoking, and physical activity level, the odds ratio (95\% confidence intervals) was higher for the presence of postprandial hyperglycemia for anthropometric indices of central fat distribution (neck circumference, waist circumference, and waist-to-height ratio) [58].

3.4. Postprandial Glucose Helps to Predict Cardiovascular Disease in Patients with Prediabetes. There has been substantial evidence found by Bergman et al. that led them to support the idea of redefining current diagnostic criteria for prediabetes with elevated $1 \mathrm{hPG}$ level. Among patients having normal glucose tolerance during the OGTT, $1 \mathrm{hPG}$ was found to be highly predictive for detecting progression to diabetes and micro- and macrovascular complications [59].

As compared with subjects with $1 \mathrm{hPG}<155 \mathrm{mg} / \mathrm{dl}$, individuals with $1 \mathrm{hPG} \geq 155 \mathrm{mg} / \mathrm{dl}$ exhibited a significantly worse cardiometabolic profile, both in the group without diabetes $(\mathrm{HbA} 1 \mathrm{c}<5.7 \%)$ and in the group with prediabetes (HbA1c 5.7-6.4\%) [60]. In the Finnish Diabetes Prevention prospective cohort study, when 504 individuals with IGT were followed up over 13 years with yearly evaluations with OGTT, FPG, and HbA1c levels, it was found that $2 \mathrm{hPG}$ was 
associated with increased risk of CVD. This supports the use of $2 \mathrm{hPG}$ in screening for prediabetes and monitoring glycaemic levels of people with prediabetes [32]. Both IFG and IGT are associated with increased cardiovascular risk as assessed by serum lipid and hsCRP levels but IGT being characterized by a more atherogenic risk profile than IFG [61]. In the EpiDREAM study, the patients from 21 countries who had IGT and IFG levels were followed up over 3.5 years, and $2 \mathrm{hPG}$ was associated with increased risk of cardiovascular events [22]. In a Chinese cross-sectional study involving prediabetic subjects, odds of developing the CVD events were associated with IGT levels rather than IFG levels [30].

\section{Conclusion}

The concept of relating the postprandial glucose as opposed to fasting glucose to coronary and cardiovascular events began as early as 1970s. This later on formed the basis of large-scale prospective studies that showed that OGTT identifies a largest number of previously undiagnosed diabetic patients with established coronary artery disease. Postprandial glucose was found to be significantly related to myocardial infarction-related mortality in nondiabetic and prediabetic patients. Additionally, studies demonstrated that controlling the postprandial hyperglycemia can prevent cardiovascular disease even in nondiabetic subjects. Biochemical markers of vascular inflammation which were hypothesized as early predictors of coronary artery disease were significantly related to postload glucose concentrations. Cardiovascular risk factors like age, body mass index, waist circumference, blood pressure, and lipid profile were significantly higher in patients with elevated postprandial glucose. The review of these studies suggests the need for reconsideration of factors on the basis of which diabetes is managed in the primary-care clinics. The patients visiting the doctor's office with their fingerstick log are usually uncertain whether they should check fasting sugars or postprandial. Some of these patients are even unsure about the duration between their meals and checking the sugar. They should be counseled about the importance of each of these blood glucose values (fasting vs. one-hour and two-hour postload glucose) and elevation of which of those values is more detrimental for their cardiovascular health. There has also been suggestion by few authors regarding revising the diagnostic criteria for prediabetes based solely on postprandial glucose for early avoidance of risk factors leading to significant cardiovascular morbidity and mortality.

\section{Conflicts of Interest}

The authors declare no conflicts of interest.

\section{Authors' Contributions}

B.M. and J.B. researched the data and wrote manuscript. J.H. reviewed and edited the manuscript.

\section{References}

[1] W. Wang, E. T. Lee, R. Fabsitz, T. K. Welty, and B. V. Howard, "Using HbAlc to improve efficacy of the American diabetes association fasting plasma glucose criterion in screening for new type 2 diabetes in American Indians: the strong heart study," Diabetes Care, vol. 25, no. 8, pp. 1365-1370, 2002.

[2] H. Cederberg, T. Saukkonen, M. Laakso et al., "Postchallenge glucose, A1C, and fasting glucose as predictors of type 2 diabetes and cardiovascular disease: a 10-year prospective cohort study," Diabetes Care, vol. 33, no. 9, pp. 2077-2083, 2010.

[3] M. de Mulder, R. M. Oemrawsingh, F. Stam, E. Boersma, and V. A. Umans, "Comparison of diagnostic criteria to detect undiagnosed diabetes in hyperglycaemic patients with acute coronary syndrome," Heart, vol. 98, no. 1, pp. 37-41, 2012.

[4] V. Gyberg, D. De Bacquer, K. Kotseva et al., "Screening for dysglycaemia in patients with coronary artery disease as reflected by fasting glucose, oral glucose tolerance test, and HbAlc: a report from EUROASPIRE IV-a survey from the European Society of Cardiology," European Heart Journal, vol. 36, no. 19, pp. 1171-1177, 2015.

[5] T. Xu, W. Liu, X. Cai et al., "Risk of coronary heart disease in different criterion of impaired fasting glucose: a meta-analysis," Medicine (Baltimore), vol. 94, Article ID e1740, 2015.

[6] K. Pyorala, "Relationship of glucose tolerance and plasma insulin to the incidence of coronary heart disease: results from two population studies in Finland," Diabetes Care, vol. 2, no. 2, pp. 131-141, 1979.

[7] K. Yano, J. S. Grove, D. M. Reed, and H. M. Chun, "Determinants of the prognosis after a first myocardial infarction in a migrant Japanese population. The Honolulu heart program," Circulation, vol. 88, no. 6, pp. 2582-2595, 1993.

[8] J. D. Curb, K. Masaki, B. L. Rodriguez et al., "Peripheral artery disease and cardiovascular risk factors in the elderly," Arteriosclerosis, Thrombosis, and Vascular Biology, vol. 16, no. 12, pp. 1495-1500, 1996.

[9] T. E. Strandberg, V. V. Salomaa, H. T. Vanhanen, V. A. Naukkarinen, S. J. Sarna, and T. A. Miettinen, "Mortality in participants and non-participants of a multifactorial prevention study of cardiovascular diseases: a 28 year follow up of the Helsinki businessmen study," Heart, vol. 74, no. 4, pp. 449-454, 1995.

[10] A. J. Orencia, M. L. Daviglus, A. R. Dyer, M. Walsh, P. Greenland, and J. Stamler, "One-hour postload plasma glucose and risks of fatal coronary heart disease and stroke among nondiabetic men and women: the Chicago heart association detection project in industry (CHA) study," Journal of Clinical Epidemiology, vol. 50, no. 12, pp. 1369-1376, 1997.

[11] M. L. Sievers, P. H. Bennett, and R. G. Nelson, "Effect of glycemia on mortality in Pima Indians with type 2 diabetes," Diabetes, vol. 48, no. 4, pp. 896-902, 1999.

[12] F. de Vegt, J. M. Dekker, H. G. Ruhé et al., "Hyperglycaemia is associated with all-cause and cardiovascular mortality in the Hoorn population: the Hoorn Study," Diabetologia, vol. 42, no. 8, pp. 926-931, 1999.

[13] J. B. Meigs, D. M. Nathan, R. B. D'Agostino Sr., and P. W. F. Wilson, "Fasting and postchallenge glycemia and cardiovascular disease risk: the Framingham offspring study," Diabetes Care, vol. 25, no. 10, pp. 1845-1850, 2002.

[14] E. J. Brunner, M. J. Shipley, D. R. Witte, J. H. Fuller, and M. G. Marmot, "Relation between blood glucose and coronary mortality over 33 years in the Whitehall study," Diabetes Care, vol. 29, no. 1, pp. 26-31, 2006. 
[15] C. Meisinger, G. Wolke, S. Brasche, G. Strube, and J. Heinrich, "Postload plasma glucose and 30-year mortality among nondiabetic middle-aged men from the general population: the ERFORT study," Annals of Epidemiology, vol. 16, no. 7, pp. 534-539, 2006.

[16] A. Nigam, M. G. Bourassa, A. Fortier, M.-C. Guertin, and J.-C. Tardif, "Fasting but not postprandial (postmeal) glycemia predicts the risk of death in subjects with coronary artery disease," Canadian Journal of Cardiology, vol. 23, no. 11, pp. 873-878, 2007.

[17] K.-L. Chien, H.-C. Hsu, T.-C. Su, M.-F. Chen, Y.-T. Lee, and F. B. Hu, "Fasting and postchallenge hyperglycemia and risk of cardiovascular disease in Chinese: the Chin-Shan community cardiovascular cohort study," American Heart Journal, vol. 156, no. 5, pp. 996-1002, 2008.

[18] N. Sarwar, T. Aspelund, G. Eiriksdottir et al., "Markers of dysglycaemia and risk of coronary heart disease in people without diabetes: reykjavik prospective study and systematic review," PLoS Medicine, vol. 7, Article ID e1000278, 2010.

[19] S. Kitada, Y. Otsuka, N. Kokubu et al., "Post-load hyperglycemia as an important predictor of long-term adverse cardiac events after acute myocardial infarction: a scientific study," Cardiovascular Diabetology, vol. 9, no. 1, p. 75, 2010.

[20] M. Shimabukuro, N. Higa, T. Asahi et al., "Impaired glucose tolerance, but not impaired fasting glucose, underlies left ventricular diastolic dysfunction," Diabetes Care, vol. 34, no. 3, pp. 686-690, 2011.

[21] I. Raz, A. Ceriello, P. W. Wilson et al., "Post hoc subgroup analysis of the HEART2D trial demonstrates lower cardiovascular risk in older patients targeting postprandial versus fasting/premeal glycemia," Diabetes Care, vol. 34, no. 7, pp. 1511-1513, 2011.

[22] S. Anand, G. Dagenais, V. Mohan et al., "Glucose levels are associated with cardiovascular disease and death in an international cohort of normal glycaemic and dysglycaemic men and women: the EpiDREAM cohort study," European Journal of Preventive Cardiology, vol. 19, no. 4, pp. 755-764, 2012.

[23] K. Tamita, M. Katayama, T. Takagi et al., "Newly diagnosed glucose intolerance and prognosis after acute myocardial infarction: comparison of post-challenge versus fasting glucose concentrations," Heart, vol. 98, no. 11, pp. 848-854, 2012.

[24] C. Furtado de Souza, M. Dalzochio, F. J. de Oliveira, J. Gross, and C. Leitão, "Glucose tolerance status is a better predictor of diabetes and cardiovascular outcomes than metabolic syndrome: a prospective cohort study," Diabetology \& Metabolic Syndrome, vol. 4, no. 1, p. 25, 2012.

[25] F. Ning, L. Zhang, J. M. Dekker et al., "Development of coronary heart disease and ischemic stroke in relation to fasting and 2-hour plasma glucose levels in the normal range," Cardiovascular Diabetology, vol. 11, no. 1, p. 76, 2012.

[26] L. Henareh and S. Agewall, "2-h postchallenge plasma glucose predicts cardiovascular events in patients with myocardial infarction without known diabetes mellitus," Cardiovascular Diabetology, vol. 11, no. 1, p. 93, 2012.

[27] G. Silbernagel, H. Sourij, T. B. Grammer et al., "Isolated postchallenge hyperglycaemia predicts increased cardiovascular mortality," Atherosclerosis, vol. 225, no. 1, pp. 194-199, 2012.

[28] B. Capaldo, P. Di Bonito, M. Iaccarino et al., "Cardiovascular characteristics in subjects with increasing levels of abnormal glucose regulation: the strong heart study," Diabetes Care, vol. 36, no. 4, pp. 992-997, 2013.

[29] M. Barzin, F. Hosseinpanah, R. Malboosbaf, F. Hajsheikholeslami, and F. Azizi, "Isolated post-challenge hyperglycaemia and risk of cardiovascular events: Tehran lipid and glucose study," Diabetes and Vascular Disease Research, vol. 10, no. 4, pp. 324-329, 2013.

[30] Z. Yang, X. Xing, J. Xiao et al., "Prevalence of cardiovascular disease and risk factors in the Chinese population with impaired glucose regulation: the 2007-2008 China national diabetes and metabolic disorders study," Experimental and Clinical Endocrinology \& Diabetes, vol. 121, no. 6, pp. 372-374, 2013.

[31] S. Kuramitsu, H. Yokoi, T. Domei et al., "Impact of postchallenge hyperglycemia on clinical outcomes in Japanese patients with stable angina undergoing percutaneous coronary intervention," Cardiovascular Diabetology, vol. 12, no. 1, p. 74, 2013.

[32] M. Lind, J. Tuomilehto, M. Uusitupa et al., "The association between $\mathrm{HbA1c}$, fasting glucose, 1-hour glucose and 2-hour glucose during an oral glucose tolerance test and cardiovascular disease in individuals with elevated risk of diabetes," PLoS One, vol. 9, no. 10, Article ID e109506, 2014.

[33] V. Ritsinger, E. Tanoglidi, K. Malmberg et al., "Sustained prognostic implications of newly detected glucose abnormalities in patients with acute myocardial infarction: longterm follow-up of the glucose tolerance in patients with acute myocardial infarction cohort," Diabetes and Vascular Disease Research, vol. 12, no. 1, pp. 23-32, 2015.

[34] A. George, R. T. Bhatia, G. L. Buchanan et al., "Impaired glucose tolerance of newly diagnosed diabetes mellitus diagnosed during admission adversely affects prognosis after myocardial infarction: an observational study," PLoS One, vol. 10, no. 11, Article ID e0142045, 2015.

[35] S. Faghihi-Kashani, F. Bonnet, N. Hafezi-Nejad et al., "Fasting hyperinsulinaemia and 2-h glycaemia predict coronary heart disease in patients with type 2 diabetes," Diabetes \& Metabolism, vol. 42, no. 1, pp. 55-61, 2016.

[36] B. Shahim, D. De Bacquer, G. De Backer et al., "The prognostic value of fasting plasma glucose, two-hour postload glucose, and $\mathrm{HbA} 1 \mathrm{c}$ in patients with coronary artery disease: a report from EUROASPIRE IV," Diabetes Care, vol. 40, no. 9, pp. 1233-1240, 2017.

[37] M. L. Nielsen, M. Pareek, M. Leósdóttir, K.-F. Eriksson, P. M. Nilsson, and M. H. Olsen, "One-hour glucose value as a long-term predictor of cardiovascular morbidity and mortality: the Malmö preventive project," European Journal of Endocrinology, vol. 178, pp. 225-236, 2018.

[38] S. Chattopadhyay, A. George, J. John, and T. Sathyapalan, "Two-hour post-challenge glucose is a better predictor of adverse outcome after myocardial infarction than fasting or admission glucose in patients without diabetes," Acta Diabetologica, vol. 55, no. 5, pp. 449-458, 2018.

[39] S. Chattopadhyay, A. George, J. John, and T. Sathyapalan, "Adjustment of the GRACE score by 2-hour post-load glucose improves prediction of long-term major adverse cardiac events in acute coronary syndrome in patients without known diabetes," European Heart Journal, vol. 39, no. 29, pp. 2740-2745, 2018.

[40] T. V. Fiorentino, E. Succurro, F. Andreozzi, A. Sciacqua, F. Perticone, and G. Sesti, "One-hour post-load hyperglycemia combined with $\mathrm{HbAlc}$ identifies individuals with higher risk of cardiovascular diseases: cross-sectional data from the CATAMERI study," Diabetes/Metabolism Research and Reviews, vol. 35, no. 2, Article ID e3096, 2019.

[41] S. Chattopadhyay, A. George, J. John, and T. Sathyapalan, "Pre-diabetes mellitus newly disgnosed after myocardial infarction adversely affects prognosis in patients without known 
diabetes," Diabetes and Vascular Disease Research, vol. 16, no. 6, pp. 489-497, 2019.

[42] Is fasting glucose sufficient to define diabetes? Epidemiological data from 20 European studies. The DECODE-study group. European Diabetes Epidemiology Group. Diabetes Epidemiology: Collaborative analysis of diagnostic criteria in Europe," Diabetologia, vol. 42, pp. 647-654, 1999.

[43] M. Hanefeld, C. Koehler, F. Schaper, K. Fuecker, E. Henkel, and T. Temelkova-Kurktschiev, "Postprandial plasma glucose is an independent risk factor for increased carotid intimamedia thickness in non-diabetic individuals," Atherosclerosis, vol. 144, no. 1, pp. 229-235, 1999.

[44] R. H. Mackey, K. Sutton-Tyrrell, P. V. Vaitkevicius et al., "Correlates of aortic stiffness in elderly individuals: a subgroup of the cardiovascular health study," American Journal of Hypertension, vol. 15, no. 1, pp. 16-23, 2002.

[45] M. Hanefeld, "Postprandial hyperglycaemia: noxious effects on the vessel wall," International Journal of Clinical Practice. Supplement, vol. 129, pp. 45-50, 2002.

[46] H. v. Bibra, T. Siegmund, A. Ceriello, M. Volozhyna, and P.-M. Schumm-Draeger, "Optimized postprandial glucose control is associated with improved cardiac/vascular function-omparison of three insulin regimens in well-controlled type 2 diabetes," Hormone and Metabolic Research, vol. 41, no. 02, pp. 109-115, 2009.

[47] L. Xu, C. Q. Jiang, T. H. Lam et al., "Impact of impaired fasting glucose and impaired glucose tolerance on arterial stiffness in an older Chinese population: the Guangzhou Biobank Cohort Study-CVD," Metabolism, vol. 59, no. 3, pp. 367-372, 2010.

[48] A. Sciacqua, S. Miceli, G. Carullo et al., "One-hour postload plasma glucose levels and left ventricular mass in hypertensive patients," Diabetes Care, vol. 34, no. 6, pp. 1406-1411, 2011.

[49] C. H. Li, J. S. Wu, Y. C. Yang, C. C. Shih, F. H. Lu, and C.-J. Chang, "Increased arterial stiffness in subjects with impaired glucose tolerance and newly diagnosed diabetes but not isolated impaired fasting glucose," The Journal of Clinical Endocrinology \& Metabolism, vol. 97, no. 4, pp. E658-E662, 2012.

[50] A. Sciacqua, R. Maio, S. Miceli et al., "Association between one-hour post-load glucose levels and vascular stiffness in essential hypertension," PLoS One, vol. 7, no. 9, Article ID e44470, 2012.

[51] I. Kowalska, M. Straczkowski, M. Szelachowska et al., "Circulating E-selectin, vascular cell adhesion molecule-1, and intercellular adhesion molecule- 1 in men with coronary artery disease assessed by angiography and disturbances of carbohydrate metabolism," Metabolism, vol. 51, no. 6, pp. 733-736, 2002.

[52] G. Derosa, A. D’Angelo, S. A. T. Salvadeo et al., "Oral glucose tolerance test effects on endothelial inflammation markers in healthy subjects and diabetic patients," Hormone and Metabolic Research, vol. 42, no. 1, pp. 8-13, 2010.

[53] E. Mah, S. K. Noh, K. D. Ballard, M. E. Matos, J. S. Volek, and R. S. Bruno, "Postprandial hyperglycemia impairs vascular endothelial function in healthy men by inducing lipid peroxidation and increasing asymmetric dimethylarginine:arginine," The Journal of Nutrition, vol. 141, no. 11, pp. 1961-1968, 2011.

[54] D. E. Maessen, N. M. Hanssen, J. L. Scheijen et al., "Postglucose load plasma a-dicarbonyl concentrations are increased in individuals with impaired glucose metabolism and type 2 diabetes: the CODAM study," Diabetes Care, vol. 38, no. 5, pp. 913-920, 2015.
[55] N. Taheri, B. Iraj, M. Amini, P. Amini, and A. Aminorroaya, "Cardiovascular risk factors in relatives of type 2 diabetics with normal glucose tolerance test and elevated one-hour plasma glucose," Endokrynologia Polska, vol. 61, no. 4, pp. 359-363, 2010.

[56] C. Bianchi, R. Miccoli, M. Trombetta et al., "Elevated 1-hour postload plasma glucose levels identify subjects with normal glucose tolerance but impaired $\beta$-cell function, insulin resistance, and worse cardiovascular risk profile: the GENFIEV study," The Journal of Clinical Endocrinology \& Metabolism, vol. 98, no. 5, pp. 2100-2105, 2013.

[57] A. Menke, K. F. Rust, and C. C. Cowie, "Diabetes based on 2-h plasma glucose among those classified as having prediabetes based on fasting plasma glucose or A1c," Diabetes and Vascular Disease Research, vol. 15, no. 1, pp. 46-54, 2018.

[58] B. Pérez-Pevida, J. M. Núñez-Córdoba, S. Romero et al., "Discriminatory ability of anthropometric measurements of central fat distribution for prediction of post-prandial hyperglycaemia in patients with normal fasting glucose: the DICAMANO Study," Journal of Translational Medicine, vol. 17, no. 1, p. 48, 2019.

[59] M. Bergman, M. Manco, G. Sesti et al., "Petition to replace current OGTT criteria for diagnosing prediabetes with the 1hour post-load plasma glucose $\geq 155 \mathrm{mg} / \mathrm{dl}(8.6 \mathrm{mmol} / \mathrm{L})$," Diabetes Research and Clinical Practice, vol. 146, pp. 18-33, 2018.

[60] T. V. Fiorentino, F. Sesti, F. Andreozzi et al., "One-hour postload hyperglycemia combined with HbAlc identifies prediabetic individuals with a higher cardio-metabolic risk burden," Atherosclerosis, vol. 253, pp. 61-69, 2016.

[61] N. Chakaroya, T. Tankova, I. Atanassova, and L. Dakoyska, "Serum lipid and hsCRP levels in prediabetes-impaired fasting glucose (IFG) and impaired glucose tolerance (IGT)," Diabetes Research and Clinical Practice, vol. 86, no. 1, pp. 56-60, 2009. 


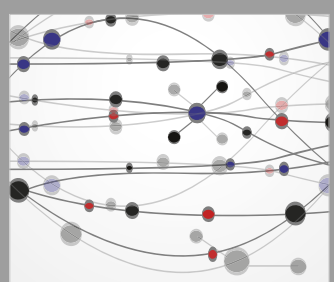

The Scientific World Journal
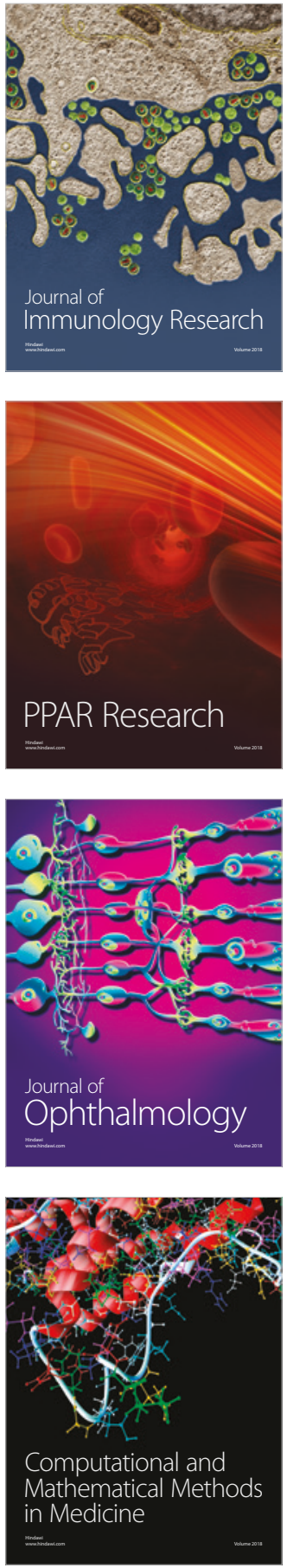

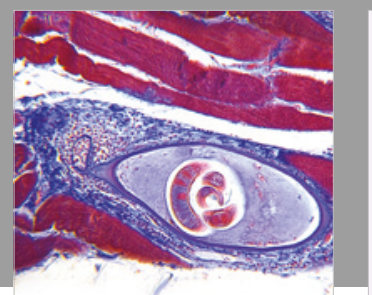

Gastroenterology Research and Practice

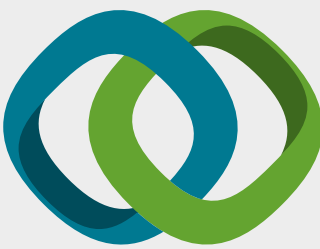

\section{Hindawi}

Submit your manuscripts at

www.hindawi.com
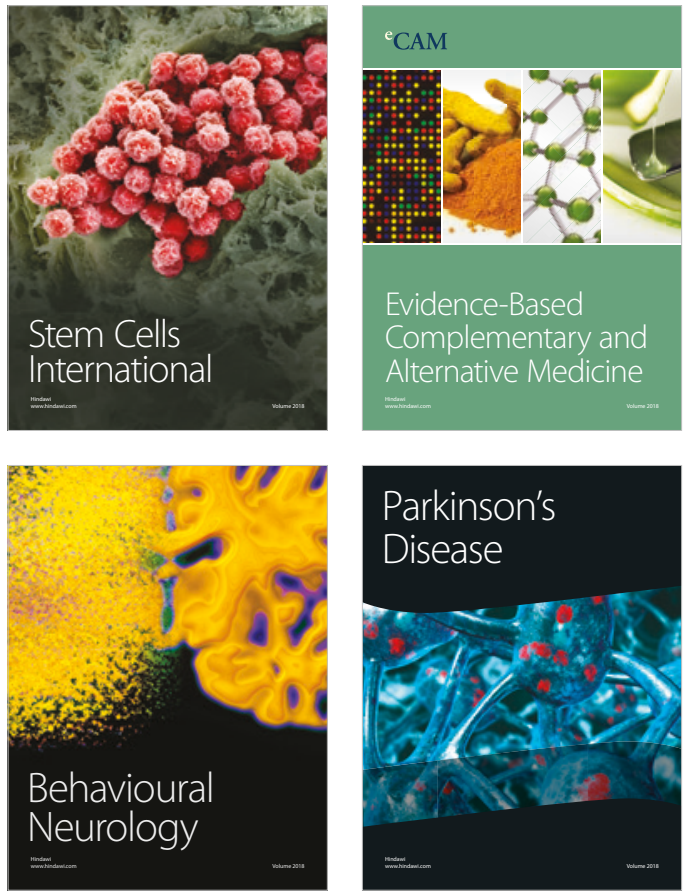

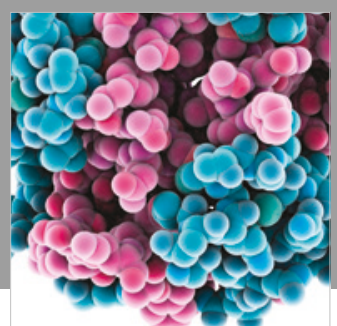

ournal of

Diabetes Research

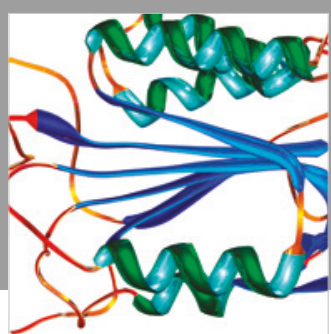

Disease Markers
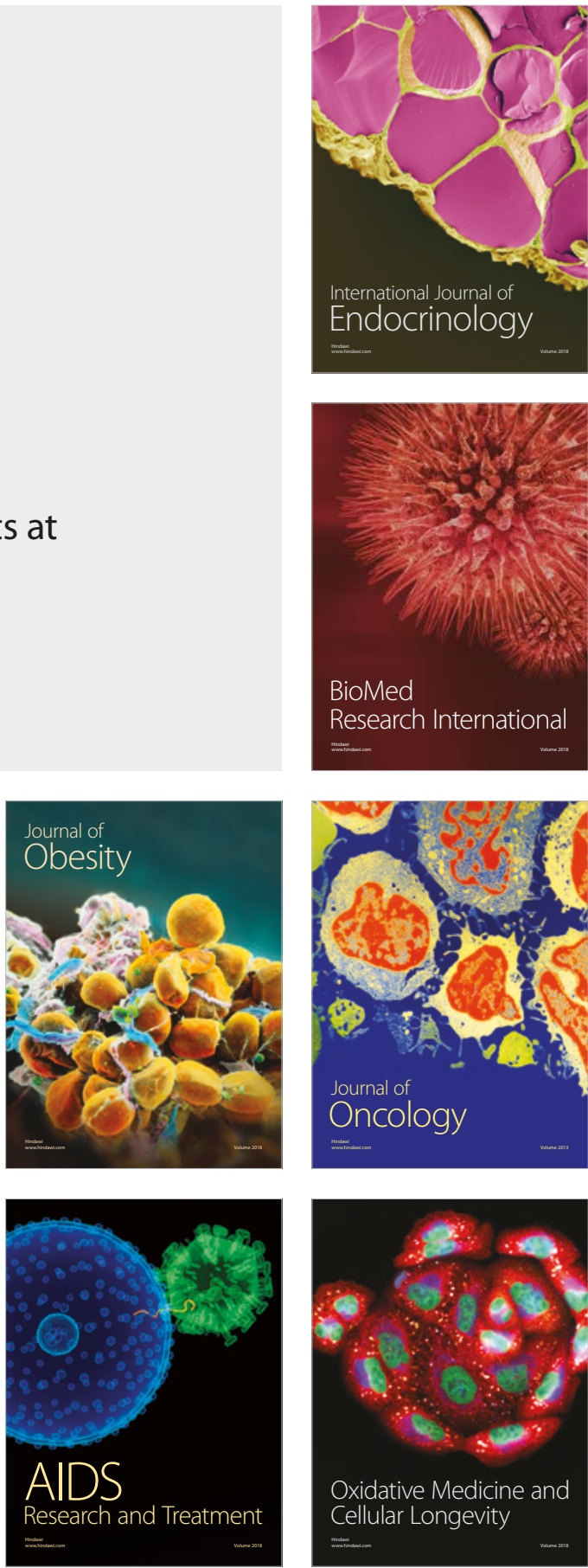